\title{
Role of Adjuvant and Posttreatment Exercise Programs in Breast Health
}

Jennifer A. Ligibel, MD, Boston, Massachusetts

Key Words

Exercise, physical activity, breast cancer, adjuvant

\begin{abstract}
Growing evidence suggests that physical activity may be an important part of survivorship care for women with a history of breast cancer. Observational evidence suggests that women who are physically active after breast cancer diagnosis have a $30 \%$ to $50 \%$ lower risk of breast cancer recurrence, breast cancer death, and overall death compared with sedentary individuals. Although randomized controlled trials have not been performed to test the ability of exercise to improve outcomes in women with early-stage breast cancer, many small intervention studies have shown the safety and potential benefits of exercise in the adjuvant and posttreatment settings. These studies have shown that physical activity can be performed safely both during and after adjuvant treatment for breast cancer, and that women who increase physical activity in these settings experience improvements in fitness, strength, quality of life, and other end points. Although more research is needed to fully define the role of exercise in breast cancer survivors, the many proven benefits of physical activity have led the American Cancer Society and American College of Sports Medicine to encourage regular participation in moderate-intensity recreational activity for most breast cancer survivors. This article reviews the growing evidence that exercise could be an important part of breast cancer survivorship, and describes current exercise guidelines for breast cancer survivors. (JNCCN 2011;9:251-256)
\end{abstract}

Accumulating evidence suggests that exercise, long known to be a fundamental part of general health maintenance, may have additional benefits for breast cancer

From Dana-Farber Cancer Institute and Harvard Medical School, Boston, Massachusetts.

Submitted September 22, 2010; accepted for publication November 4, 2010.

The author has disclosed that she has no financial interests, arrangements, or affiliations with the manufacturers of any products discussed in this article or their competitors.

Correspondence: Jennifer A. Ligibel, MD, Dana-Farber Cancer Institute, 44 Binney Street, Boston, MA 02115.

E-mail: jligibel@partners.org survivors. Observational evidence suggests that women who exercise after breast cancer diagnosis have lower rates of recurrence and death compared with sedentary individuals. A growing number of exercise intervention studies show that increased physical activity after diagnosis leads to improvements in quality of life, fatigue, and body image, amongst other end points, and preliminary work shows that exercise can lead to improvements in biomarkers linked to breast cancer prognosis.

\section{Observational Evidence}

Dozens of studies have examined the relationship between physical activity and breast cancer risk, with most showing a lower risk of breast cancer in active women. ${ }^{1-3} \mathrm{~A}$ recent review of 62 cohort and case-control studies published before 2007 shows a reduction of breast cancer risk in active women in $76 \%$ of studies, with an average risk reduction of $25 \%$ to $30 \%$ in the most versus least active women. ${ }^{4}$

More recently, physical activity has also been associated with better outcomes in women who develop early-stage breast cancer. Four recent, large, prospective studies have shown that women who are physically active after breast cancer diagnosis have an improved prognosis compared with less-active women (Table 1). The Nurses' Health Study examined the relationship between physical activity after diagnosis and rates of breast cancer recurrence and death in a cohort of 2987 women diagnosed with stage I through IIIa breast cancer. ${ }^{5}$ After 96 months median follow-up, patients who engaged in more than 9 metabolic equivalent task (MET) hours per week of physical activity, equivalent to walking at an average pace for 3 hours per week, had a 50\% lower risk of breast cancer recurrence, breast cancer death, and all-cause mortality than women who were inactive (engaging in less than an hour of moderate-intensity 
Ligibel

\begin{tabular}{|c|c|c|c|c|c|}
\hline Study & $\mathbf{N}$ & $\begin{array}{l}\text { Timing of } \\
\text { Exercise } \\
\text { Assessment }\end{array}$ & $\begin{array}{l}\text { Median } \\
\text { Follow-up }\end{array}$ & $\begin{array}{l}\text { Number of } \\
\text { Events }\end{array}$ & Results \\
\hline $\begin{array}{l}\text { Nurses' Health } \\
\text { Study }(\mathrm{NHS})^{5}\end{array}$ & 2987 & $\begin{array}{l}\geq 2 \text { y after } \\
\text { diagnosis }\end{array}$ & $96 \mathrm{mo}$ & $\begin{array}{l}463 \text { deaths; } \\
370 \text { recur- } \\
\text { rences; } \\
280 \text { breast } \\
\text { cancer deaths }\end{array}$ & $\begin{array}{l}\text { Patients who exercised } \geq 9 \text { MET-hours of } \\
\text { exercise per week had a significant } \\
\text { reduction in recurrence, breast cancer death, } \\
\text { and overall death: HR, } 0.57(0.38-0.85)\end{array}$ \\
\hline $\begin{array}{l}\text { Women's Healthy } \\
\text { Eating and Living } \\
\text { Study (WHEL) }\end{array}$ & 1490 & $\begin{array}{l}\text { Within } 4 \mathrm{y} \\
\text { of diagnosis }\end{array}$ & $80 \mathrm{mo}$ & $\begin{array}{l}236 \text { total } \\
\text { events; } 135 \\
\text { deaths }\end{array}$ & $\begin{array}{l}\text { Patients who consumed } \geq 5 \text { vegetable/fruit } \\
\text { servings per day and had } 9 \text { MET-hours per } \\
\text { week of activity had a significant reduction } \\
\text { in mortality: HR, } 0.56(0.31-0.98)\end{array}$ \\
\hline $\begin{array}{l}\text { Collaborative } \\
\text { Women's Longev- } \\
\text { ity Study (CWLS) }\end{array}$ & 4482 & $\begin{array}{l}\geq 5 \text { y after } \\
\text { diagnosis }\end{array}$ & $66 \mathrm{mo}$ & $\begin{array}{l}109 \text { breast } \\
\text { cancer deaths }\end{array}$ & $\begin{array}{l}\text { Patients who exercised at least } 2.8 \text { MET- } \\
\text { hours per week had a significant reduction } \\
\text { in breast cancer death: HR, } 0.58(0.45-0.75)\end{array}$ \\
\hline $\begin{array}{l}\text { Health, Eating, } \\
\text { Activity and } \\
\text { Lifestyle Study } \\
(\text { HEAL) }\end{array}$ & 668 & $\begin{array}{l}3 \text { y after } \\
\text { diagnosis }\end{array}$ & $72 \mathrm{mo}$ & 164 deaths & $\begin{array}{l}\text { Patients who exercised at least } 9 \text { MET-hours } \\
\text { per week had a significant reduction in } \\
\text { breast cancer death: HR, } 0.33(0.15-0.73)\end{array}$ \\
\hline $\begin{array}{l}\text { Life After Cancer } \\
\text { Study (LACE) }\end{array}$ & 1870 & $\begin{array}{l}\text { Within } 39 \text { mo } \\
\text { of diagnosis }\end{array}$ & $87 \mathrm{mo}$ & $\begin{array}{l}225 \text { recurrenc- } \\
\text { es; } 102 \text { breast } \\
\text { cancer deaths }\end{array}$ & $\begin{array}{l}\text { No relationship between exercise and breast } \\
\text { cancer recurrence or related mortality in } \\
\text { multivariate analysis ( } P \text { for trend }=.36 \text { and } \\
.26 \text {, respectively) }\end{array}$ \\
\hline
\end{tabular}

Abbreviations: HR, hazard ratio; MET, metabolic equivalent task.

recreational activity per week). The decreased risk of recurrence and death associated with exercise was seen in both pre- and postmenopausal patients and was independent of body mass index.

Similar findings were reported in the Collaborative Women's Longevity Study (CWLS) ${ }^{6}$ and the Health, Eating, Activity and Lifestyle (HEAL) study. ${ }^{7}$ In CWLS, women expending more than 2.8 MET-hours per week had lower breast cancer mortality compared with women with lower levels of activity (hazard ratio [HR], 0.65; 95\% CI, 0.39-1.08 for 2.8-7.9 MET-hours per week; and HR, 0.59; 95\% CI, 0.35-1.01 for 8.0-20.9 MET-hours per week), and every additional 5 MET-hours per week of moderate activity was associated with a $15 \%$ decrease in the risk of breast cancer death $(P$ for trend $=.03)$. In the HEAL study, women who engaged in at least 9 METhours per week of activity had an HR for mortality of 0.33 (95\% CI, 0.15-0.73). ${ }^{7}$ Despite the limited power from the small number of events, the study also suggested that, compared with women who maintained a constant level of activity, those who decreased activity after diagnosis had an increased risk of death (HR, 3.95; 95\% CI, 1.45-10.50) and those who increased activity had a decreased risk of death (HR, 0.55; 95\% CI, 0.22-1.38).
Finally, physical activity in combination with a healthy diet was shown to be associated with improved survival in the Women's Healthy Eating and Living Study. ${ }^{8}$ Women who both consumed 5 or more servings of fruits and vegetables per day and completed at least 540 MET-minutes of physical activity each week (equivalent to walking at a moderate pace for 3 hours per week) were found to have a mortality of $4.8 \%$, compared with $10 \%$ in participants who had either low physical activity or low fruit and vegetable intake, and $11.5 \%$ in participants who had low physical activity and low fruit and vegetable intake.

However, not all studies evaluating the relationship between physical activity and breast cancer outcomes show better survival among physically active patients. The Life After Cancer Epidemiology (LACE) study ${ }^{9}$ (Table 1) reported a trend toward a lower risk of recurrence and breast cancer mortality among women with higher levels of physical activity in univariate analysis ( $P$ for trend $=.05$ and .07 , respectively, for highest vs. lowest level of hours per week of moderate physical activity), but these associations were attenuated in multivariate analysis adjusting for known prognostic factors $(P$ for trend $=.36$ and .26, respectively). These discrepancies highlight 
the need for randomized trials testing the ability of increased exercise after cancer diagnosis to improve prognosis in women with early-stage breast cancer.

\section{Exercise Intervention Studies in Breast Cancer Survivors}

Dozens of small-to-moderate-sized interventional studies have tested the feasibility and potential benefits of exercise in the adjuvant setting, ${ }^{10-12}$ although none have been large enough to examine the impact of physical activity on breast cancer outcomes. Studies have examined exercise interventions both during and after adjuvant treatment. End points have included various measures, such as quality of life, fitness, fatigue, body image, anthropometric, and biomarkers. Both supervised and unsupervised exercise programs have been tested, and exercise modalities studied have included walking, cycling, yoga, strength training, and rowing.

Interest in this area has grown exponentially in the past few years. A full review of the many exercise intervention studies is beyond the scope of this manuscript but can be found in several recent publications. The American College of Sports Medicine (ACSM) published a comprehensive review of exercise intervention studies in breast cancer populations as part of a recent roundtable discussion of exercise in cancer survivors. ${ }^{10}$ The review included data from 54 randomized controlled trials of exercise in patients with breast cancer: 22 in the adjuvant setting and 32 in the posttreatment setting. The authors found consistent evidence that exercise could be performed safely in both the adjuvant and posttreatment settings. Exercise led to significant improvements in aerobic fitness and strength in both settings and led to increased flexibility and physical functioning in the posttreatment setting. A moderate level of evidence also suggested that exercise led to improvements in quality of life, anxiety, depression, fatigue, body image, body size, and body composition in breast cancer survivors, although findings were not always consistent.

Two smaller reviews of exercise intervention studies in patients with breast cancer included metaanalyses to explore the magnitude of benefits conferred by exercise in breast cancer populations. A Cochrane review summarized data from 9 studies of aerobic and strength-training interventions dur- ing adjuvant chemotherapy and/or radiation among patients with breast cancer. ${ }^{11}$ The studies included a total of 452 patients, with individual studies ranging in size from 10 to 123 patients. Although study end points were varied, the authors were able to conduct meta-analyses of cardiorespiratory fitness, fatigue, and weight measures. Across the studies, participants in the exercise interventions experienced significant improvements in cardiorespiratory fitness compared with control subjects (standard mean difference [SMD], 0.66; 95\% CI, 0.2-1.12). Metaanalyses for fatigue and weight gain showed nonsignificant improvements in the exercise groups (SMD, -0.12 ; 95\% CI, $-0.37-0.13$, and SMD, $-1.11 ; 95 \%$ $\mathrm{CI},-2.44-0.22$, respectively). Given heterogeneity among studies, conclusions regarding the impact of exercise on other end points, such as quality of life, anxiety, and depression, were limited.

In another review, McNeely et al..$^{12}$ analyzed randomized controlled exercise studies with quality of life, fitness, or physical functioning end points. The authors identified 14 studies in the adjuvant and posttreatment periods that met their criteria, involving 717 participants. Pooled data from these studies showed that exercise led to significant improvements in quality of life as assessed by the Functional Assessment of Cancer Therapy-General (FACT-G) and Breast (FACT-B) scales (weighted mean difference [WMD], 4.58; 95\% CI, 0.35-8.80, and WMD, 6.62; 95\% CI, 1.21-12.03, respectively). Exercise also led to improvements in cardiorespiratory fitness, as measured by peak oxygen consumption (WMD, 3.39; 95\% CI, 1.67-5.10); in physical functioning (SMD, $0.84 ; 95 \% \mathrm{CI}, 0.36-1.32$ ); and in fatigue (SMD, 0.46; 95\% CI, 0.23-0.70), although significant improvements in fatigue were only seen in studies conducted after the completion of adjuvant therapy.

Thus, physical activity has been shown to have several benefits for patients with breast cancer both during and after adjuvant therapy. No clear consensus exists on the "best" type of physical activity, but benefits have been seen with several different moderateintensity exercise modalities, and incorporating this type of activity seems to be safe throughout the adjuvant and survivorship periods. The small size of these intervention studies does not allow conclusions to be made regarding the impact of increasing physical activity on breast cancer prognosis. However, although more work is needed to fully define its role in improv- 
Ligibel

ing breast cancer outcomes, regular physical activity should be encouraged given its many other general and breast cancer-specific health benefits.

\section{Special Considerations: Lymphedema}

Breast cancer survivors have long been cautioned against lifting heavy objects after undergoing axillary surgery, given concerns that it could increase the risk of lymphedema. This recommendation has led many women to avoid upper-body strength training exercise. Recent evidence has called these recommendations into question, instead suggesting that slowly progressive strength training exercise many be beneficial for breast cancer survivors.

Ahmed et al. ${ }^{13}$ conducted a randomized trial of supervised upper- and lower-body strength training in 45 breast cancer survivors at risk of lymphedema. Participants met with an ACSM-certified instructor for training sessions, and started upper-body exercise with no or half-pound weights for each exercise. None of the women randomized to the strength training group developed lymphedema, and selfreport arm symptoms were the same in the exercise and control groups. In a subsequent study evaluating a strength training intervention in women with preexisting lymphedema, Schmitz et al. ${ }^{14}$ found that the women randomized to the exercise intervention reported significantly fewer lymphedema exacerbations and overall improvements in arm symptoms compared with those in the control group. Notably, all of the women wore a custom-fitted compression garment on the affected arm and were monitored by a lymphedema specialist throughout the study.

Additionally, a recent Cochrane review of 24 upper-body exercise intervention studies in breast cancer survivors suggested that exercise training improved range of motion without conferring an increased risk of lymphedema. ${ }^{15}$ Although these studies suggest that upper-body strength training is safe in breast cancer survivors, most of the intervention studies reported used supervised, tightly regulated strength training programs.

\section{Biologic Mechanisms}

The biologic mechanisms through which physical activity could impact breast cancer risk and pathogenic potential remain unclear. Current hypotheses focus on exercise-induced changes in sex steroid, metabolic, and inflammatory pathways that have been linked to breast cancer risk and outcomes. Sex steroids have long been linked to breast cancer risk, with higher levels of estrogen and testosterone associated with increased breast cancer rates in postmenopausal women. ${ }^{16,17}$ More recently, metabolic and inflammatory pathways have also been linked to breast cancer risk and outcomes. A recent meta-analysis has shown an increased risk of breast cancer in patients with type II diabetes, ${ }^{18}$ and several recent studies have shown an increased risk of cancer recurrence in patients without diabetes who have higher levels of fasting insulin at breast cancer diagnosis. ${ }^{19,20} \mathrm{El}$ evated levels of insulin-like growth factor 1 (IGF-1) have been associated with an increased risk of premenopausal breast cancer, ${ }^{21}$ and several other adipose-related hormones, such as leptin and adiponectin, have been implicated in breast cancer risk and outcomes. ${ }^{22,23}$ Inflammatory mediators such as tumor necrosis factor $\alpha$ and interleukin 6 (IL-6) have been linked to breast cancer risk, ${ }^{24}$ and C-reactive protein (CRP) has been associated with an increased risk of breast cancer diagnosis and recurrence in patients with early-stage disease. ${ }^{25}$

Physical activity has been shown to impact many of these sex steroid, metabolic, and inflammatory pathways, either directly or through affecting body composition. Observational data suggest that physical activity is associated with lower levels of insulin, leptin, IL-6, and CRP, ${ }^{26,27}$ and exercise intervention studies in women at risk of breast cancer and in breast cancer survivors have shown that increases in physical activity can lead to decreases in serum levels of insulin, IGF-1, estradiol, and CRP. ${ }^{28-31}$ Animal models also show that physical activity can lower rates of breast cancer development in rat models, ${ }^{32}$ and also provide some early information regarding the impact of physical activity on inflammatory and metabolic pathways in rat breast tissues. ${ }^{33}$ However, little information is currently available about the impact of physical activity on breast cancer at the cellular level in humans, and much work remains to be done to elucidate the biologic pathways through which exercise could lead to improvements in breast cancer prognosis. 
Adjuvant and Posttreatment Exercise

Table 2 American College of Sports Medicine Exercise Guidelines for Breast Cancer Survivors ${ }^{10}$

- Avoid inactivity

- Return to normal levels of activity as soon as possible after surgery

- Consult with a physician before beginning an exercise program

- Formal exercise testing is not required before beginning a moderate-intensity exercise program unless cardiovascular or other comorbidities exist

- Try to meet aerobic activity recommendations for general population ( $\geq 150$ minutes per week of aerobic activity)

- Upper-body exercise

- Evaluation for upper extremity morbidity is recommended before starting an exercise program

> Start with supervised strength-training sessions

> Start with low or no resistance

- Although no upper limit of weight exists to which breast cancer survivors can progress, weight should be increased very slowly

- Lower resistance or stop specific exercises if symptoms of lymphedema occur

- Be aware of fracture risk if undergoing treatment with agents that can lower bone density

\section{Clinical Recommendations and Conclusions}

A growing body of observational evidence suggests that women who are physically active after breast cancer diagnosis have better outcomes than more sedentary individuals. Although these data cannot be regarded as definitive proof that physical activity improves breast cancer prognosis, multiple exercise intervention studies show that physical activity after breast cancer diagnosis leads to improvements in quality of life, fitness, strength, and other health outcomes. In light of these data, the American Cancer Society ${ }^{34}$ and ACSM $^{10}$ have issued physical activity guidelines for cancer survivors. Both groups recommend that cancer survivors incorporate moderate-intensity physical activity into their daily lives. The recently released ACSM guidelines ${ }^{10}$ (Table 2 ) encourage cancer survivors to comply with the Physical Activity Guidelines for Americans, which recommend at least 150 minutes of moderate-intensity aerobic exercise each week. The guidelines also conclude that moderate-intensity aerobic exercise, such as walking, is safe for most patients with cancer and do not recommend more involved evaluation or exercise testing, such as a treadmill stress test, for most patients before beginning a moderate exercise program.

The ACSM guidelines also conclude that strength training seems to be beneficial for cancer survivors, but caution that women with a history of breast cancer should consider a supervised program for upper-body strength training. ACSM also recently developed a cancer exercise training certification process to provide exercise professionals with a curriculum for exercise concerns specific to patients with cancer. As this certification program is disseminated to the many exercise professionals throughout the United States, it will provide patients and medical professionals with a referral network to help breast cancer survivors increase physical activity after cancer diagnosis. Finally, many other cancer-specific exercise programs are being developed and implemented in local communities throughout the United States, such as the LIVESTRONG Program, a joint effort by the Lance Armstrong Foundation and the YMCA, which provides exercise programs for cancer survivors throughout the United States.

Growing evidence suggests that physical activity, a mainstay of general health maintenance, may be especially important for breast cancer survivors. Exercise has been shown to have several benefits for breast cancer survivors, and could even lead to better outcomes in patients with early-stage disease. Regular moderate-intensity exercise is recommended for breast cancer survivors, and a growing number of programs and community-based resources are being developed to help women increase activity after breast cancer diagnosis.

\section{References}

1. Thune I, Ferberg A. Physical activity and cancer risk: doseresponse and cancer, all sites and site specific. Med Sci Sports Exerc 2001;33:S530-550. 
2. McTiernan A, Kooperberg C, White E, et al. Recreational physical activity and the risk of breast cancer in postmenopausal women. JAMA 2003;290:1331-1336.

3. Maruti S, Willett W, Feshanich D, et al. A prospective study of agespecific physical activity and premenopausal breast cancer. J Natl Cancer Inst 2008;100:728-737.

4. Friedenreich CM, Cust AE. Physical activity and breast cancer risk: impact of timing, type and dose of activity and population subgroup effects. Br J Sports Med 2008;42:636-647.

5. Holmes M, Chen W, Feskanich D, et al. Physical activity and survival after breast cancer diagnosis. JAMA 2005;293:2479-2486.

6. Holick C, Newcomb P, Trentham-Dietz A, et al. Physical activity and survival after diagnosis of invasive breast cancer. Cancer Epidemiol Biomarkers Prev 2008;17:379-386.

7. Irwin $M$, Smith $A, M c T i e r n a n ~ A$, et al. Influence of pre- and postdiagnosis physical activity on mortality in breast cancer survivors: the Health, Eating, Activity, and Lifestyle study. J Clin Oncol 2008;26:3958-3964.

8. Pierce J, Stefanick M, Flatt $S$, et al. Greater survival after breast cancer in physically active women with high vegetable-fruit intake regardless of obesity. J Clin Oncol 2007;17:2345-2351.

9. Sternfeld B, Weltzien E, Quesenberry CP Jr, et al. Physical activity and risk of recurrence and mortality in breast cancer survivors: findings from the LACE study. Cancer Epidemiol Biomarkers Prev 2009;18:87-95.

10. Schmitz KH, Courneya KS, Matthews C, et al. American College of Sports Medicine roundtable on exercise guidelines for cancer survivors. Med Sci Sports Exerc 2010;42:1409-1426.

11. Markes M, Brockow $T$, Resch $K$. Exercise for women receiving adjuvant therapy for breast cancer. Cochrane Database Syst Rev 2006;CD005001.

12. McNeely M, Campbell K, Rowe B, et al. Effects of exercise on breast cancer patients and survivors: a systematic review and metaanalysis. CMAJ 2006;175:34-41.

13. Ahmed RL, Thomas W, Yee D, Schmitz KH. Randomized controlled trial of weight training and lymphedema in breast cancer survivors. J Clin Oncol 2006;24:2765-2772.

14. Schmitz KH, Ahmed RL, Troxel A, et al. Weight lifting in women with breast-cancer-related lymphedema. N Engl J Med 2009;361:664-673.

15. McNeely ML, Campbell K, Ospina M, et al. Exercise interventions for upper-limb dysfunction due to breast cancer treatment. Cochrane Database Syst Rev 2010;CD005211.

16. Key T, Appleby P, Barnes I, et al. Endogenous sex hormones and breast cancer in postmenopausal women: reanalysis of nine prospective studies. J Natl Cancer Inst 2002;94:606-612.

17. Berrino F, Pasanisi P, Bellati C, et al. Serum testosterone levels and breast cancer recurrence. Int J Cancer 2005;113:499-502.

18. Larsson S, Mantzoros C, Wolk A. Diabetes mellitus and risk of breast cancer: a meta-analysis. Int J Cancer 2007;121:856-862.

19. Goodwin P, Ennis M, Pritchard K, Trudeau M. Fasting insulin and outcome in early-stage breast cancer: results of a prospective cohort study. J Clin Oncol 2002;20:42-51.

20. Pollak M, Chapman J, Shepherd L, et al. Insulin resistance, estimated by serum C-peptide level, is associated with reduced event-free survival for postmenopausal women in NCIC CTG MA.14 adjuvant breast cancer trial. J Clin Oncol 2006;24:524.

21. Hanksinson S, Willett W, Colditz G, et al. Circulating concentrations of insulin-like growth factor-1 and risk of breast cancer. Lancet 1998;351:1393-1396.

22. Rose DP, Komninou D, Stephenson GD. Obesity, adipocytokines, and insulin resistance in breast cancer. Obes Rev 2004;5:153-165.

23. Mantzoros C, Petridou E, Dessypris N, Chavelas C. Adiponectin and breast cancer risk. J Clin Endocrinol Metab 2004;89:1102_ 1107.

24. Neilson H, Friedenreich C, Brockton N, Millikan R. Physical activity and postmenopausal breast cancer: proposed biologic mechanisms and areas for future research. Cancer Epidemiol Biomarkers Prev 2009;18:11-27.

25. Pierce BL, Ballard-Barbash R, Bernstein L, et al. Elevated biomarkers of inflammation are associated with reduced survival among breast cancer patients. J Clin Oncol 2009;27:3437-3444.

26. Mathur N, Pedersen B. Exercise as a means to control systemic, low grade inflammation. Mediators Inflamm 2008;2008:109502.

27. Irwin $M$, McTiernan $A$, Bernstein $L$, et al. Relationship of obesity and physical activity with C-peptide, leptin, and insulinlike growth factors in breast cancer survivors. Cancer Epidemiol Biomarkers Prev 2005;14:2881-2888.

28. Ligibel JA, Giobbie-Hurder A, Olenczuk D, et al. Impact of a mixed strength and endurance exercise intervention on levels of adiponectin, high molecular weight adiponectin and leptin in breast cancer survivors. Cancer Causes Control 2009;20:15231528 .

29. Irwin M, Varma K, Alvarez-Reeves M, et al. Randomized controlled trial of aerobic exercise on insulin and insulin-like growth factors in breast cancer survivors: the Yale Exercise and Survivorship study. Cancer Epidemiol Biomarkers Prev 2009;18:306-313.

30. Frank L, Sorensen B, Yasui Y, et al. Effects of exercise on metabolic risk variables in overweight postmenopausal women: a randomized clinical trial. Obes Res 2005;13:615-625.

31. Campbell PT, Campbell KL, Wener MH, et al. A yearlong exercise intervention decreases CRP among obese postmenopausal women. Med Sci Sports Exerc 2009;41:1533-1539.

32. Thompson H. Effects of physical activity and exercise on experimentally-induced mammary carcinogenesis. Breast Cancer Res Treat 1997;46:135-141.

33. Jiang W, Zhu Z, Thompson HJ. Effects of physical activity and restricted energy intake on chemically induced mammary carcinogenesis. Cancer Prev Res (Phila) 2009;2:338-344.

34. Doyle C, Kushi L, Byers T, et al. Nutrition and physical activity during and after cancer treatment: an American Cancer Society guide for informed choices. CA Cancer J Clin 2006;56:323-353. 